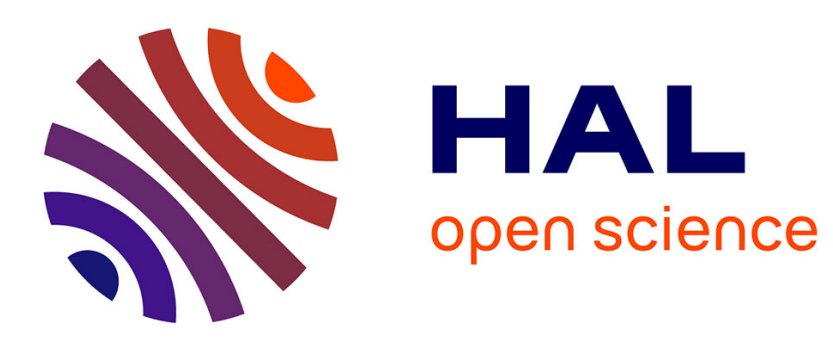

\title{
Optimal Cooperative Checkpointing for Shared High-Performance Computing Platforms
}

Thomas Hérault, Yves Robert, Aurélien Bouteiller, Dorian Arnold, Kurt B Ferreira, George Bosilca, Jack Dongarra

\section{- To cite this version:}

Thomas Hérault, Yves Robert, Aurélien Bouteiller, Dorian Arnold, Kurt B Ferreira, et al.. Optimal Cooperative Checkpointing for Shared High-Performance Computing Platforms. [Research Report] RR-9109, INRIA. 2017, pp.1-20. hal-01621295

\section{HAL Id: hal-01621295 \\ https://hal.inria.fr/hal-01621295}

Submitted on 23 Oct 2017

HAL is a multi-disciplinary open access archive for the deposit and dissemination of scientific research documents, whether they are published or not. The documents may come from teaching and research institutions in France or abroad, or from public or private research centers.
L'archive ouverte pluridisciplinaire HAL, est destinée au dépôt et à la diffusion de documents scientifiques de niveau recherche, publiés ou non, émanant des établissements d'enseignement et de recherche français ou étrangers, des laboratoires publics ou privés. 


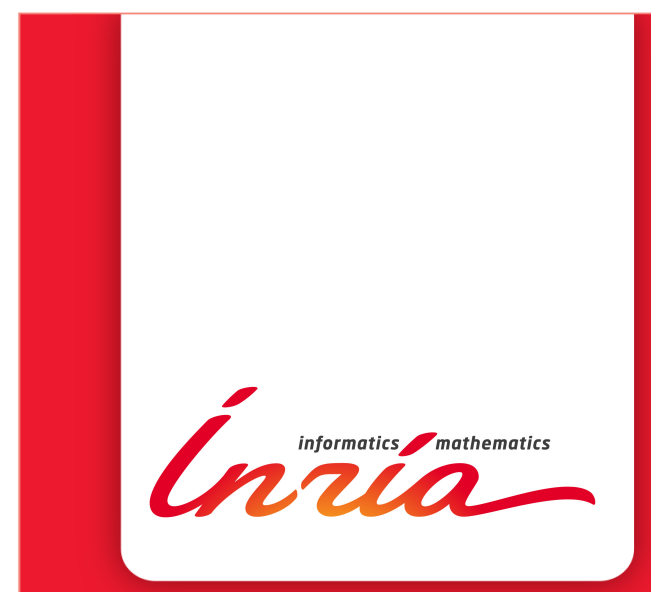

\section{Optimal Cooperative} Checkpointing for Shared High-Performance Computing Platforms

Thomas Herault, Yves Robert, Aurelien Bouteiller, Dorian Arnold, Kurt B. Ferreira, George Bosilca, Jack Dongarra

RESEARCH REPORT

$\mathbf{N}^{\circ} 9109$

October 2017

Project-Team ROMA 



\title{
inzián
}

\section{Optimal Cooperative Checkpointing for Shared High-Performance Computing Platforms *}

\author{
Thomas Herault $^{\dagger}$, Yves Robert ${ }^{\ddagger \dagger}$, Aurelien Bouteiller ${ }^{\dagger}$, Dorian \\ Arnold $^{\S}$, Kurt B. Ferreira ${ }^{\mathbb{I}}$, George Bosilca ${ }^{\dagger}$, Jack Dongarra ${ }^{\dagger}$ \\ Project-Team ROMA
}

Research Report nº 9109 - October 2017 - 20 pages

\begin{abstract}
In high-performance computing environments, input/output (I/O) from various sources often contend for scare available bandwidth. Adding to the $\mathrm{I} / \mathrm{O}$ operations inherent to the failure-free execution of an application, I/O from checkpoint/restart (CR) operations (used to ensure progress in the presence of failures) places an additional burden as it increases $\mathrm{I} / \mathrm{O}$ contention, leading to degraded performance. In this work, we consider a cooperative scheduling policy that optimizes the overall performance of concurrently executing CR-based applications which share valuable $\mathrm{I} / \mathrm{O}$ resources. First, we provide a theoretical model and then derive a set of necessary constraints needed to minimize the global waste on the platform. Our results demonstrate that the optimal checkpoint interval as defined by Young/Daly, while providing a sensible metric for a single application, is not sufficient to optimally address resource contention at the platform scale. We therefore show that combining optimal checkpointing periods with $\mathrm{I} / \mathrm{O}$ scheduling strategies can provide a significant improvement on the overall application performance, thereby maximizing platform throughput. Overall, these results provide critical analysis and direct guidance on checkpointing large-scale workloads in the presence of competing $\mathrm{I} / \mathrm{O}$ while minimizing the impact on application performance.
\end{abstract}

Key-words: checkpoint, I/O contention, shared platform, scheduling policy, cooperative checkpoint.

* Based in part upon work supported by the National Science Foundation under Grant Number 1564133, "Toward Extreme Scale Fault-Tolerance: Exploration Methods, Comparative Studies and Decision Processes".

$\dagger$ University of Tennessee Knoxville, USA

$\ddagger$ Ecole Normale Superieure de Lyon \& INRIA, France

$\S$ Emory University, Atlanta, GA, USA

$\mathbb{I}$ Center for Computing Research, Sandia National Laboratory, USA

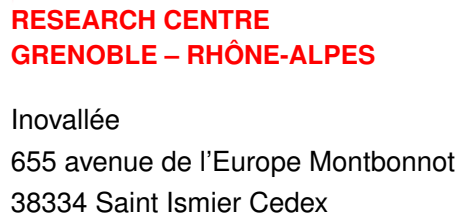




\section{Stratégies de checkpoint coopératives sur plates-formes partagées}

Résumé : Ce rapport s'intéresse aux plates-formes de calcul scientifique partagées, i.e., sur lesquelles s'exécutent simultanément plusieurs classes d'applications. Celles-ci sont en compétition pour l'accès aux ressources d'entrées-sorties, à la fois pour leurs opérations de base et pour prendre leurs checkpoints. Nous proposons un modèle et analysons plusieurs stratégies de prise de checkpoints, à période fixe ou dépendant de l'application, avec ou sans interférence, bloquante ou non. Nous déterminons une borne inférieure sur la fraction de temps nécessairement perdue par la plateforme pour toute stratégie de checkpoint/redémarrage, et nous montrons expérimentalement que notre stratégie coopérative obtient des performances très proches de cette borne. Dans notre stratégie coopérative, les périodes de checkpoint des applications ne sont pas nécessairement celles calculées par la formule de Young/Daly, car la bande passante disponible ne permet pas toujours de les mettre en oeuvre, et certaines applications ont nécessairement une période plus longue (et donc sous-optimale). Nous donnons les résultats d'un ensemble de simulations menées avec des ensembles de paramètres pour les applications et les plates-formes qui correspondent à des scénarios actuels et prospectifs.

Mots-clés : résilience, checkpoint, optimisation I/O, stratégie d'ordonnancement. 


\section{Introduction}

Space-sharing high-performance computing (HPC) platforms for the concurrent execution of multiple parallel applications is the prevalent usage pattern in today's HPC centers. In fact, spacesharing in this fashion is more common than capability workloads that span the entire platform [1]. Furthermore, while computational nodes are dedicated to a particular application instance, the interconnect links and storage partition are typically shared amongst application instances. Therefore, without careful consideration, network and storage contention can reduce individual application and overall system performance [2].

On these platforms, checkpoint/restart (CR) is the most common strategy employed to protect applications from underlying faults and failures. Generally, CR periodically outputs snapshots (i.e. checkpoints) of its global, distributed state to some stable storage device. When an application failure occurs, the last stored checkpoint is retrieved and used to restart the application. Typically, concurrently executing applications independently decide when to take their own checkpoints.

There are two widely-used approaches to determine when an application should commit a checkpoint: (i) using a fixed checkpoint period (typically one or a few hours) for each application; and (ii) using platform and application-specific metrics to determine its optimal checkpoint period. In the second approach, the well-known Young/Daly formula [3, 4] yields an application optimal checkpoint period, $\sqrt{2 \mu C}$ seconds, where $C$ is the time to commit a checkpoint and $\mu$ the application Mean Time Between Failures (MTBF) of the platform. In most cases, $\mu=\frac{\mu_{\text {ind }}}{q}$, where $q$ is the number of processors enrolled by the application and $\mu_{\text {ind }}$ is the MTBF of an individual processor [5]. Therefore, both $\mu$ and $C$ in the Young/Daly formula are application-dependent, and optimal periods can be quite different over the application spectrum.

Independent $\mathrm{CR}$ of concurrent application instances can incur significant resource wastage, because they lead to an inefficient usage of an already scarce resource, namely available I/O bandwidth [6]. There are two major reasons for this:

- Application-CR I/O contention: On many systems, the I/O subsystem does not have enough available usable bandwidth to meet the requirements of the concurrent application workloads [6]. This congestion is expected to worsen going forward with the increased prevelance of data intensive workflows in HPC. Let $\beta_{\text {tot }}$ be the total filesystem I/O bandwidth. Concurrently executing applications typically perform regular (non-CR) I/O operations throughout their execution, so that only a fraction $\beta_{\text {avail }}$ of the total bandwidth remains available for checkpoints. This fraction may be insufficient, particularly when some applications perform intensive non-checkpoint I/O and others may write very large checkpoints.

- CR-CR I/O contention: Most importantly, there is a high probability of overlapping CR activity amongst concurrent application instances. Consider the simple case where two applications of same size checkpoint simultaneously a file of the same size. Each will be assigned half the fraction $\beta_{\text {avail }}$ to checkpoint, therefore the commits will take twice as long. Such interferences can severely decrease application efficiency and overall platform throughput $^{1}$.

In this work, we develop and investigate a cooperative CR scheduling strategy for concurrently executing HPC applications. Our objective is to assess the impact of such interferences, and to design scheduling algorithms that optimize I/O bandwidth availability for CR activity. Using these cooperative algorithms, applications checkpoint sequentially, with a dynamic, priority-dependent frequency dictated by a cooperative scheduler. When enough I/O bandwidth is available, each application checkpoints with its optimal, Young/Daly, period. However, when I/O bandwidth is scarce, our scheduling algorithm provides an optimal checkpoint period that maximizes overall platform throughput. This cooperative checkpoint process is calculated such that there is no I/O interference and minimal re-work to be done when failures occur.

Therefore, the main contributions of this paper are the following:

- Development of a model allowing for the quantification of the I/O interference of checkpoint-

\footnotetext{
${ }^{1}$ When the expected checkpoint commit time used to compute the optimal checkpoint interval differs from the actual checkpoint commit time, effciency will decrease.
} 
ing applications sharing a common underlying I/O substrate.

- Investigation of the costs of various I/O-aware scheduling strategies through both steadystate analysis as well as detailed simulations.

- A detailed survey of a number scheduling strategies: from oblivious algorithms similar to those currently deployed on many large-scale platforms, to ones which exploit application knowledge in an effort to minimize the total system waste by scheduling the application with the most critical I/O needs.

The rest of the paper is organized as follows. Our model is described in Section 2, followed by a description of the various scheduling strategies in Section 3. Section 4 presents a theoretical analysis of the model under a steady-state scenario, and provides a lower bound of the optimal platform waste. Section 5 describes the discrete event simulator used to quantitatively compare the different scheduling strategies. Section 6 presents the results of the simulation, providing guidance on the necessary I/O bandwidth for current and future systems. This work concludes with related works described in Section 7, followed by a summary and future directions outlined in Section 8.

\section{Model}

Computational Platform Model In this work, we consider a shared platform comprised of computational nodes, storage resources in the form of a parallel file system (PFS), and a network that interconnects the nodes and storage resources. Applications are scheduled on the platform by a job scheduler such that computational nodes are space-shared (dedicated) amongst concurrent application instances. However, the I/O subsystem is time-shared (contended) amongst application instances (i.e. multiple applications performing $\mathrm{I} / \mathrm{O}$ simultaneously result in a perapplication reduction in commit speed). Without loss of generality, we consider a straightforward linear interference model in which the global throughput remains constant and is evenly shared among contending applications, proportional to their size ${ }^{2}$.

Application Workload Model Applications can vary in size (computational node count), duration, memory footprint and I/O requirements. Application $I / O$ entails loading an input file at startup, performing regular $\mathrm{I} / \mathrm{O}$ operations during their main execution phase and storing an output file at completion. Because applications are long-running, (typically, several hours or days) and the platform is failure-prone, applications are protected using coordinated CR that incurs periodic $C R I / O$.

To model these behavioral variations with minimal parameters, we make the following simplifying assumptions:

- There is a large number of applications, but only a small number of application classes, i.e., sets of applications with similar sizes, durations, footprints and I/O needs;

- Excluding initialization and finalization I/O, an application's regular (non-CR) I/O operations are evenly distributed over its makespan;

- Job makespans are known a priori. This allows us to ignore all other sources of job disturbance except $\mathrm{C} / \mathrm{R}$ overheads.

We use specific numbers and characteristics of application classes based on documented production workloads, such as those provided in the APEX workflows report on the Cielo platform [7]. To avoid the side effects induced by hundreds of completely identical jobs, we use a normal distributions for job durations with a mean equal to original APEX value and small (20\%) standard deviation. In the rest of the paper, we use the term job to denote a specific application instance, and application class to denote a set of applications with similar characteristics.

\footnotetext{
${ }^{2} \mathrm{~A}$ more adversarial interference model can be substituted, if needed.
} 
Checkpoint Period and I/O Interference Both application computation and CR generate $\mathrm{I} / \mathrm{O}$ requests. In both cases, activity is scheduled using an I/O scheduling algorithm (see Section 3 ). As described above, steady-state application I/O is regular. However, CR I/O periodicity, $P$, depends upon the CR policy being used. In our model, applications either checkpoint using an application-defined periodicity or using Young and Daly's [3, 4] optimal checkpoint period detailed in Section 1. As stated previously, the parameters in this formula are dependent upon application features (checkpoint dataset size) and platform features (system reliability and I/O bandwidth). For fixed, application-defined periods, a common heuristic is to take a checkpoint every hour capping the worst case amount of lost work at one hour. In the reminder of this paper we will refer to the two variants as Fixed (with a 1 hour period unless otherwise specified) and Daly.

Traditionally, when a job $J_{i}$ of class $A_{i}$ completes a checkpoint, its next checkpoint is scheduled to happen $P_{i}-C_{i}$ instants later (and the first checkpoint is set at date $P_{i}$ ). With potential CR $\mathrm{I} / \mathrm{O}$ interference, the checkpoint commit may last longer than $C_{i}$, and setting the appropriate checkpointing period can be challenging. Additionally, I/O scheduling algorithms that try to mitigate I/O interference can impose further $\mathrm{CR} \mathrm{I/O}$ delays. In other words, the traditional strategy of scheduling subsequent checkpoints at $P_{i}-C_{i}$ yields the desired checkpointing period $P_{i}$ only in interference-free scenarios. CR I/O delays (induced by interferences or scheduling delays) dilate the checkpoint duration to $C_{\text {dilated }}$, and the effective period differs from the desired period by the difference $C_{\text {dilated }}-C_{i}$. Section 3 discusses how each I/O scheduling algorithm handles this discrepancy.

Job Scheduling Model To evaluate the scheduling policies, we consider a finite segment, typically lasting a few days, of a representative schedule where the computing resource usage by each application instance (job) in each class remains nearly constant. Of course, with varying job execution times, we cannot enforce a fixed proportion of each application class at every instant. However, we ensure the proper proportion is enforced on average throughout the schedule execution. Similarly, we enforce that at every instant during the finite segment, at least $98 \%$ of the nodes are enrolled for the execution. This allows us to compare actual (simulated) performance with the theoretical performance of a co-scheduling policy that optimizes the steady-state I/O behavior of the job portfolio, assuming that all processors are used. We shuffle and simultaneously present all jobs to the scheduler, which uses a simple, greedy first-fit algorithm. We resubmit failed jobs with a new wall-time equal to the fraction that remained when the last checkpoint commit started. In this case, input I/O becomes recovery I/O; output I/O is unmodified.

The Formal Model We consider a set $\mathcal{A}$ of $|\mathcal{A}|$ applications classes $A_{1}, \ldots A_{|\mathcal{A}|}$ that execute concurrently on a platform with $\mathcal{N}$ nodes. Application class $A_{i}$ specifies:

- $n_{i}$ : the number of jobs in $A_{i}$,

- $q_{i}$ : the number of nodes used by each job in $A_{i}$,

- $P_{i}$ : the checkpoint period of each job in $A_{i}$, and

- $C_{i}$ and $R_{i}$ : the checkpoint and recovery durations for each job in $A_{i}$ when there is no interference with other I/O operations.

Jobs inherit their characteristics from their classes. To simplify notations, for a job $J_{j}$, we use $q_{j}, P_{j}, C_{j}$ and $R_{j}$ to denote respectfully the number of nodes, checkpoint period, and checkpoint and recovery durations of the application class to which $J_{j}$ belongs. We let $P_{D a l y}\left(J_{j}\right)=\sqrt{2 C_{j} \mu_{j}}$ be the Daly period $[3,4]$ of a job $J_{j}$, where $\mu_{j}=\frac{\mu_{\text {ind }}}{q_{j}}$ and $\mu_{\text {ind }}$ is the MTBF of an individual processor [5]. At each instance, we schedule as many jobs as possible. Jobs that are subject to failures are restarted at the head of the scheduling queue, as to restart immediately on the same compute nodes previously used (in most cases, only one node has failed and is replaced by a hot spare). 


\section{$3 \quad$ I/O Scheduling Algorithms}

In this section, we present the application I/O scheduling algorithms used in this study. The first algorithm, Oblivious, represents the status-quo in which I/O activities are scheduled independently and may incur slowdowns due to I/O contention. The second algorithm, Ordered, coordinates I/O activity to eliminate interference: I/O operations are scheduled in a First-Come-First-Serve (FCFS) fashion and only one I/O operation executes at any given time, while other $\mathrm{I} / \mathrm{O}$ requests are blocked until their turn comes. The third algorithm, Ordered-NB, is similar except that jobs that are waiting for the I/O token to checkpoint continue working until their turn comes. Lastly, we propose our heuristic, Least-Waste, which improves on Ordered-NB by giving the I/O token to the I/O operation that will minimize system waste. Note that unlike the blocking approaches (Oblivious and Ordered), non-blocking optimizations (Ordered-NB and Least-Waste) may require application code refactoring.

\subsection{Oblivious I/O Scheduling}

In Oblivious I/O scheduling, jobs are executed to fill-up the system based on processor availability, and their I/O workload (including CR activities) are not coordinated by the system. Instead, jobs use the parallel file system assuming they are the sole user - with no modifications made to their access patterns to accommodate for possible interference. Researchers have observed that concurrent I/O resource access can decrease the I/O bandwidth observed [8]. Under the conditions of an under-provisioned I/O substrate, our model gives each I/O stream a decrease in bandwidth linearly proportional to the number of competing operations. We account for the additional delays imposed by this decreased available bandwidth as waste. Since subsequent checkpoints are scheduled to start after $P_{i}-C_{i}$, and delays may result in checkpoint commit times longer than $C_{i}$, the resultant checkpoint period may be longer than $P_{i}$. This is consistent with a trivial $\mathrm{I} / \mathrm{O}$ policy that does not consider potential contention.

\subsection{Blocking Ordered FCFS I/O Scheduling}

A simple optimization to the Oblivious scheme is to favor one jobs' I/O over all others. While the overall throughput may remain unchanged (given an efficient filesystem implementation), the favored job completes its I/O workload faster (i.e., in time $C_{i}$ for a job of class $A_{i}$ ). In the Ordered scheme, I/O requests are performed sequentially, in request arrival order. Jobs with outstanding $\mathrm{I} / \mathrm{O}$ requests are blocked until their requests are completed.

Assuming a favorable linear interference model, a simple workload with two jobs can show the potential advantage of the Ordered over Oblivious strategy. If the two jobs simultaneously request $\mathrm{I} / \mathrm{O}$ transfers of similar data volume, $V$, in the Oblivious strategy, both jobs take $\frac{V}{\frac{\beta_{\text {avail }}}{2}}$ time to complete their I/O. In the Ordered strategy, the first scheduled job takes only $\frac{V}{\beta_{\text {avail }}}$, while the second job waits $\frac{V}{\beta_{\text {avail }}}$ before its own I/O starts, but then executes at full available bandwidth completing in $\frac{2 V}{\beta_{\text {avail }}}$. Reducing $\mathrm{I} / \mathrm{O}$ interference reduces the average $\mathrm{I} / \mathrm{O}$ completion time (although fairness may be decreased). Once again, however, observed checkpoint durations may increase past $C_{i}$, due to I/O scheduling wait time, and the checkpointing period may be, on average, larger than the desired $P_{i}$.

\subsection{Non-Blocking Ordered-NB FCFS I/O Scheduling}

The previous strategy trades the cost of I/O interferences for idle time, as jobs perform a blocking (idle) wait for the $\mathrm{I} / \mathrm{O}$ token. If the application developer can refactor the program code to continue computing while awaiting $\mathrm{I} / \mathrm{O}$ request completions, it becomes possible to replace otherwise idle wait time with useful computation. In the Ordered- $N B$ algorithm, when the previous checkpoint ends at time $t_{\text {now }}$, a tentative time for the next checkpoint is set at $t_{\text {req }}=t_{\text {now }}+P_{i}-C_{i}$. At time $t_{r e q}$, a non-blocking $\mathrm{I} / \mathrm{O}$ request is made to request the $\mathrm{I} / \mathrm{O}$ token - the $\mathrm{I} / \mathrm{O}$ token is still scheduled 
FCFS according to request arrival time. The job continues its computation until the scheduler informs it that the $\mathrm{I} / \mathrm{O}$ token is available. At this point, the job must generate its checkpoint data as soon as possible (or after a short synchronization ${ }^{3}$ ). In most applications, the granularity of the work is small enough for a simple approach to be efficient: applications can use existing APIs in SCR [9] or FTI [10] to regularly poll if a checkpoint should be taken at this time. In this work, we assume that this re-synchronization cost is negligible relative to the checkpoint commit duration. Postponing checkpoint I/O increases a job's exposure to failures. However, if the job successfully commits the postponed checkpoint, upon a subsequent failure, the job would restart from the time at which the postponed checkpoint was taken, not at $t_{r e q}$ - a fact that may mitigate the increased risk exposure when compared to Ordered and Oblivious algorithms.

\subsection{Variants}

The periods $P_{i}$ of the checkpointing requests are input parameters to the three strategies Oblivious, Ordered and Ordered-NB. In Section 5, we instantiate each strategy with two variants. The first variant uses a fixed checkpointing period for each job, while the second variant uses the Daly period of each job.

\subsection{Least-Waste Algorithm}

Finally, our Least-Waste algorithm further refines the Ordered-NB algorithm by issuing the I/O token to the job whose $\mathrm{I} / \mathrm{O}$ request minimizes the total expected waste (explained hereafter), rather than simply based on request arrival order. Given the time-dependent nature of this decision, the selection may not be a global optimum, but only an approximation given currently available information about the system status. The Least-Waste algorithm assumes that jobs issue checkpointing requests according to their Daly period ${ }^{4}$. For each I/O scheduling decision, at time $t$ (when a previous $\mathrm{I} / \mathrm{O}$ operation completes), we consider a pool of $r+s$ candidates from two different categories:

- Category IO-Candidate $\mathcal{C}_{I O}$ : Jobs $J_{i}, 1 \leq i \leq r$ with an (input, output or recovery) I/O request of length $v_{i}$ seconds and enrolls $q_{i}$ processors. $J_{i}$ initiated its $\mathrm{I} / \mathrm{O}$ request $d_{i}$ seconds ago and has been idle for $d_{i}$ seconds.

- Category Ckpt-Candidate $\mathcal{C}_{C k p t}$ : Jobs $J_{i}, r+1 \leq i \leq r+s$, with a checkpoint duration of $C_{i}$ seconds and enrolls $q_{i}$ processors. $J_{i}$ took its last checkpoint $d_{i}$ seconds ago and keeps executing until the $\mathrm{I} / \mathrm{O}$ token is available for a new checkpoint. Since $J_{i}$ is a candidate, $d_{i} \geq P_{\text {Daly }}\left(J_{i}\right)$

If we select job $J_{i}$ to perform I/O, the expected waste $W_{i}$ incurred to the other $r+s-1$ candidate jobs in $\mathcal{C}_{I O} \cup \mathcal{C}_{C k p t}$ is computed as follows. Assume first that $J_{i} \in \mathcal{C}_{I O}$. Then $J_{i}$ will use the $\mathrm{I} / \mathrm{O}$ resource for $v_{i}$ seconds.

- Every other job $J_{j} \in \mathcal{C}_{I O}$ will stay idle for $v_{i}$ additional seconds, hence its waste $W_{i}(j)$ is

$$
W_{i}(j)=q_{j}\left(d_{j}+v_{i}\right)
$$

since there are $q_{j}$ processors enrolled in $J_{j}$ that remain idle for $d_{j}+v_{i}$ seconds. Note that for $J_{j} \in \mathcal{C}_{I O}$, the waste $W_{i}(j)$ is deterministic.

- Every job $J_{j} \in \mathcal{C}_{C k p t}$ will continue executing for $v_{i}$ additional seconds, hence will be exposed to the risk of a failure that will strike within $v_{i} / 2$ seconds on average. The probability of such a failure is $v_{i} / \mu_{j}$, where $\mu_{j}=\mu_{\text {ind }} / q_{j}$. With this probability, the $q_{j}$ processors will have to recover and re-execute $d_{j}+v_{i} / 2$ seconds of work, hence the waste $W_{i}(j)$ is

$$
W_{i}(j)=\frac{v_{i}}{\mu_{j}} q_{j}\left(R_{j}+d_{j}+\frac{v_{i}}{2}\right)=\frac{v_{i}}{\mu_{\text {ind }}} q_{j}^{2}\left(R_{j}+d_{j}+\frac{v_{i}}{2}\right)
$$

\footnotetext{
${ }^{3}$ In user-level checkpointing, the job typically finishes its current computing block before generating its checkpoint data.

${ }^{4}$ Fixed checkpointing makes little sense in the Least-Waste strategy, it is designed to optimize checkpoint frequencies across all jobs.
} 
where $R_{j}$ is the recovery time for $J_{j}$. Note that for $J_{j} \in \mathcal{C}_{C k p t}$, the waste $W_{i}(j)$ is probabilistic.

Altogether, the expected waste $W_{i}$ incurred to the other $r+s-1$ candidate jobs is

$$
W_{i}=\sum_{J_{j} \in \mathcal{C}_{I O}, j \neq i} W_{i}(j)+\sum_{J_{j} \in \mathcal{C}_{C k p t}} W_{i}(j)
$$

We obtain

$$
\begin{aligned}
W_{i}= & v_{i} \times\left(\sum_{1 \leq j \leq r, j \neq i} q_{j}\left(d_{j}+v_{i}\right)\right. \\
& \left.+\sum_{r+1 \leq j \leq r+s} \frac{q_{j}^{2}}{\mu_{\text {ind }}}\left(R_{j}+d_{j}+\frac{v_{i}}{2}\right)\right)
\end{aligned}
$$

Assume now that the selected job $J_{i} \in \mathcal{C}_{C k p t}$. Then $J_{i}$ will use the I/O resource for $C_{i}$ seconds instead of $v_{i}$ seconds for $J_{i} \in \mathcal{C}_{I O}$. We directly obtain the counterpart of Equation (1) for its waste $W_{i}$ :

$$
\begin{aligned}
W_{i}= & C_{i} \times\left(\sum_{1 \leq j \leq r} q_{j}\left(d_{j}+C_{i}\right)\right. \\
& \left.+\sum_{r+1 \leq j \leq r+s, j \neq i} \frac{q_{j}^{2}}{\mu_{\text {ind }}}\left(R_{j}+d_{j}+\frac{C_{i}}{2}\right)\right)
\end{aligned}
$$

Finally, we select the job $J_{i} \in \mathcal{C}_{I O} \cup \mathcal{C}_{C k p t}$ whose waste $W_{i}$ is minimal.

\section{Lower Bound}

We now derive a lower bound for optimal platform waste. When we assess the performance of the scheduling algorithms presented in Section 3, we also compare their relative performance to this lower bound (in Section 6).

We envision a (theoretical) scenario in which the platform operates in steady-state, a constant number of jobs per application class spanning the entire platform. We also assume that the

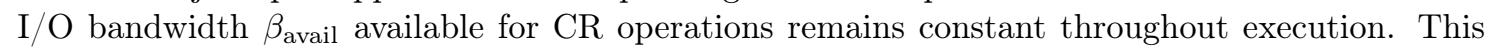
amounts to ignoring initial input and final output I/O operations, or more precisely, to assuming these operations span the entire execution of the jobs. Without this assumption, we would need to account for job durations; this renders the steady-state analysis intractable. Given above, we determine the optimal checkpointing period for each application class with the objective to minimize the total waste of the platform; or equivalently, to maximize the total throughput of the platform. To complicate this analysis, these optimal periods may not be achievable, hence we derive a lower bound of the optimal waste.

In steady-state operation, there are $n_{i}$ jobs of class $A_{i}$, each using $q_{i}$ nodes, and with checkpoint time $C_{i}$. Because we orchestrate checkpoints to avoid CR-CR interferences, we have $C_{i}=\frac{\text { size }_{i}}{\beta_{\text {avail }}}$, where size $_{i}$ denote the size of the checkpoint file of all jobs of class $A_{i}$. The waste of a job is the ratio of time the job spends doing resilience operations by the time it does useful work. The time spent performing resilience operations include the time spent during each period to checkpoint; and in case of failure, the time to rollback to the previous checkpoint and the time to recompute lost work. We can express the waste $W_{i}$ of a job $J_{i}$ of class $A_{i}$ that checkpoints with period $P_{i}$ as follows [5]:

$$
W_{i}=W_{i}\left(C_{i}\right)=\frac{C_{i}}{P_{i}}+\frac{q_{i}}{\mu}\left(\frac{P_{i}}{2}+R_{i}\right)
$$

Let $W$ be the waste of the platform. We define this as the weighted arithmetic mean of the $W_{i}$ for all applications, where each application is weighted by the number of computing nodes it uses:

$$
W=\sum_{i} \frac{n_{i} q_{i}}{\mathcal{N}} W_{i}
$$


In the absence of $\mathrm{I} / \mathrm{O}$ constraints, the checkpointing period can be minimized for each job independently. Indeed, the optimal period for a job of class $A_{i}$ is obtained by minimizing $W_{i}$ in Equation (3).

Differentiating and solving

$$
\frac{\delta W_{i}}{\delta P_{i}}=-\frac{C_{i}}{P_{i}^{2}}+\frac{q_{i}}{2 \mu}=0
$$

we readily derive that

$$
P_{i}=\sqrt{2 \frac{\mu}{q_{i}} C_{i}}=\sqrt{2 \mu_{i} C_{i}}
$$

where $\mu_{i}$ is the MTBF of class $A_{i}$ applications, and we retrieve the Daly period $P_{i}=P_{\text {Daly }}\left(J_{i}\right)$.

However, I/O constraints may impose the use of sub-optimal periods. If each job of class $A_{i}$ checkpoints in time $C_{i}$ during its period $P_{i}$ (hence without any contention), it uses the I/O device during a fraction $\frac{C_{i}}{P_{i}}$ of the time. The total usage fraction of the $\mathrm{I} / \mathrm{O}$ device is $F=\sum_{i} \frac{n_{i} C_{i}}{P_{i}}$ and cannot exceed 1. Therefore, we have to solve the following optimization problem: find the set of values $P_{i}$ that minimize $W$ in Equation (4) subject to the $\mathrm{I} / \mathrm{O}$ constraint:

$$
F=\sum_{i} \frac{n_{i} C_{i}}{P_{i}} \leq 1
$$

Hence the optimization problem is to minimize:

$$
W=\sum_{i} \frac{n_{i} q_{i}}{\mathcal{N}}\left(\frac{C_{i}}{P_{i}}+\frac{q_{i}}{\mu}\left(\frac{P_{i}}{2}+R_{i}\right)\right)
$$

subject to Equation (6). Using the Karush-Kuhn-Tucker conditions [11], we know that there exists a nonnegative constant $\lambda$ such that

$$
-\frac{\delta W}{\delta P_{i}}=\lambda \frac{\delta F}{\delta P_{i}}
$$

for all $i$. We derive that

$$
\frac{n_{i} q_{i} C_{i}}{\mathcal{N} P_{i}^{2}}-\frac{n_{i} q_{i}^{2}}{2 \mu \mathcal{N}}=-\lambda \frac{n_{i} C_{i}}{P_{i}^{2}}
$$

for all $i$. This leads to:

$$
P_{i}=\sqrt{\frac{2 \mu \mathcal{N}}{q_{i}^{2}}\left(\frac{q_{i}}{\mathcal{N}}+\lambda\right) C_{i}}
$$

for all $i$. Note that when $\lambda=0$, Equation (8) reduces to Equation (5).

Because of the I/O constraint in Equation (6), we choose for $\lambda$ the minimum value such that Equation (6) is satisfied. If $\lambda \neq 0$, this will lead to periods $P_{i}$ larger than the optimal value of Equation (5). Note that there is no closed-form expression for the minimum value of $\lambda$, it has to be found numerically.

Altogether, we state our main result:

Theorem 1. In the presence of $I / O$ constraints, the optimal checkpoint periods are given by Equation (8), where $\lambda$ is the smallest non-negative value such that Equation (6) holds. The total platform waste is then given by Equation (7).

The optimal periods may not be achievable, because Equation (6) is a necessary, but not sufficient condition. Even though the total I/O bandwidth is not exceeded, meaning there is enough capacity to take all the checkpoints at the given periods, we would still need to orchestrate these checkpoints into an appropriate, periodic, repeating pattern. In other words, we only have a lower bound of the optimal platform waste. 


\section{Simulation Framework}

We use discrete event simulations to evaluate the performance of the proposed approaches. Our simulations ${ }^{5}$ are instantiated by a set of initial conditions that define a set of application classes, the distribution of resource usage between application classes, and the main characteristics of the platform on which application instances will execute.

High level parameters Application classes are characterized by: initial input and output sizes, checkpoint size, quantity of work to execute, number of nodes to use, volume of I/O to execute during job makespan, and job compute time.

Platforms are characterized by the number of nodes, a system Mean Time Between Failures, and an aggregated I/O subsystem bandwidth that is shared among the nodes. For simplicity, we assume symmetric read and write filesystem bandwidths, hence $C_{i}=R_{i}$ for each application class, $A_{i}$.

A simulation first randomly selects a list of jobs that are instances of the different application classes. This list is ordered by job priority (i.e., arrival time for our FCFS algorithms) and constrained by two parameters: the minimum simulated time to consider, and the relative proportion of platform resources used by each application class (based on the APEX report [7]). As an example, we consider the subset of application classes given by the APEX workflows report for the subset of application classes of LANL (EAP, LAP, Silverton and VPIC), simulated as is executed on the Cielo supercomputer, for a minimal execution time of 60 days. A simulation will randomly instantiate one of the four classes, assigning a work duration uniformly distributed between $0.8 \mathrm{w}$ and $1.2 w$, where $w$ is the typical walltime specified for the chosen application class, and count the resource allocated for this application class, until 1.) the simulated execution would necessarily run for at least 2 months, and 2.) resources used by the selected class is within $1 \%$ of the target goal of the representative workload percentage defined in the APEX workflows report (see Table 1).

In addition to the jobs list, we generate a set of node failure times according to an exponential distribution with the specified MTBF. At the chosen times, we randomly choose which of the nodes fail. These jobs list and failure times constitute the initial conditions of a simulation.

Job Scheduling We compute a job schedule (start and end times for all jobs in the list) using a simple first-fit strategy considering: job characteristics, job priority and resource availability. We simulate online scheduling; whenever a job ends at a date different than the initially planned end date (because of failures, or because the I/O interference made the job extend after its planned end date), the schedule is amended by re-scheduling all jobs that were not started yet.

Execution Simulation Once a job is started, it executes its initial input. It then, 1.) executes some work for a certain period before it, and 2.) checkpoints. These two steps are repeated until all planned work is executed, after which the final output is executed by the job, before it ends. At any time during the execution, a node hosting the job may be subject to a failure (according to the pre-computed failure times and location). When that happens, the job is terminated and a new job is added to the list of jobs to schedule. That new job represents the restart of the failed one; it has similar characteristics except its initial input corresponds to the restart size, and its work time corresponds to the remaining work from the last successful checkpoint. To reflect a common job scheduling policy on shared platforms, restarted jobs are set to the highest priority, maximizing their chances of obtaining an immediate allocation and continuing what was the original (failed) jobs execution.

Interference Models Our simulations implement each of the interference models and avoidance strategies defined in Section 3: for Oblivious-Fixed and Oblivious-Daly, interfering I/O and checkpoints get a portion of the available aggregated bandwidth proportional to the number of nodes they use, and inversely proportional to the number of nodes involved for all jobs doing I/O;

\footnotetext{
${ }^{5}$ The simulator is publicly available from https://github.com/SMURFSorg/InterferingCheckpoints.
} 


\begin{tabular}{|l|c|c|c|c|}
\hline Workflow & EAP & LAP & Silverton & VPIC \\
\hline Workload percentage & 66 & 5.5 & 16.5 & 12 \\
\hline Work time (h) & 262.4 & 64 & 128 & 157.2 \\
\hline Number of cores & 16384 & 4096 & 32768 & 30000 \\
\hline Initial Input (\% of memory) & 3 & 5 & 70 & 10 \\
\hline Final Output (\% of memory) & 105 & 220 & 43 & 270 \\
\hline Checkpoint Size (\% of memory) & 160 & 185 & 350 & 85 \\
\hline
\end{tabular}

Table 1: LANL Workflow Workload from the APEX Workflows report.

for Ordered-Fixed and Ordered-Daly, I/O requests and checkpoints are ordered in a first-come first-served basis, and when they are selected, obtain the full bandwidth; for Ordered-NB-Fixed and Ordered-NB-Daly, I/O requests and checkpoints are served in order, but the simulation adds all the time waiting for a checkpoint to start as progress in the computation for the job; and for Least-Waste, the same is implemented, but $\mathrm{I} / \mathrm{O}$ is ordered to minimize the waste in Equations (1) and (2).

Note that in the scheduled I/O methods (Ordered-NB and Least-Waste), initial inputs and final outputs are blocking (the job cannot progress during the $\mathrm{I} / \mathrm{O}$ until it is served), but checkpoints are non-blocking, which means that if a failure hits the job, it may have to re-execute from a checkpoint far in its past if it has not been granted access to the filesystem for an extended period of time.

Method of statistics collection from simulations We compute the distribution of performance of each strategy using the Monte Carlo method: a large set of initial conditions (at least a thousand) is randomly chosen, and we simulate the execution of the system over each element of this set for each strategy. Since simulations for the various scheduling strategies have different initial conditions (including job mix), it would be misleading to compare simple averages of the time spent doing useful work (or time wasted) across simulation instances. Instead, we collect performance statistics over a fixed length segment of each simulation and extract and compare waste/work ratios that can be compared appropriately. The segment excludes the first and last days of the simulation: during the first day, jobs may be synchronized artificially because a subset starts at the same date, and during the last day, large amounts of resources may not be used as new jobs are not added to the workload. For each aggregate measurement, we compute and show mean, first and ninth decile, and first and third quartile statistics.

\section{Results}

\subsection{LANL APEX Simulation Workflows on Cielo}

We consider the workload from LANL found in the APEX Workflows report [7] that consists of four applications classes: EAP, LAP, Silverton and VPIC. The main characteristics of these classes are reported in Table 1. We simulate the behavior of these applications on the Cielo Platform. Cielo was a 1.37 Petaflops capability system operated from 2010 to 2016 at the Los Alamos National Laboratory. It consisted of 143,104 cores, $286 \mathrm{~TB}$ of main memory, and a parallel filesystem with a theoretical maximum capacity of $160 \mathrm{~GB} / \mathrm{s}$. Cielo was chosen for this initial analysis due to the availability of the aforementioned workflows report, something not available for other platforms. In later sections, we consider similar workloads on a more modern platform.

The baseline in this comparison comprises a set of simulations with no faults, checkpoints, nor $\mathrm{I} / \mathrm{O}$ interference. For these simulations, we selected a 60-day execution segment, and computed the resources used by the jobs during this period, i.e. the total time each node spent on (non-CR) $\mathrm{I} / \mathrm{O}$ and computation in a failure-free environment.

For the I/O scheduling techniques presented in Section 3, we compute the resource waste as the total time nodes spend not progressing jobs. In the figures presented, we represent the performance 


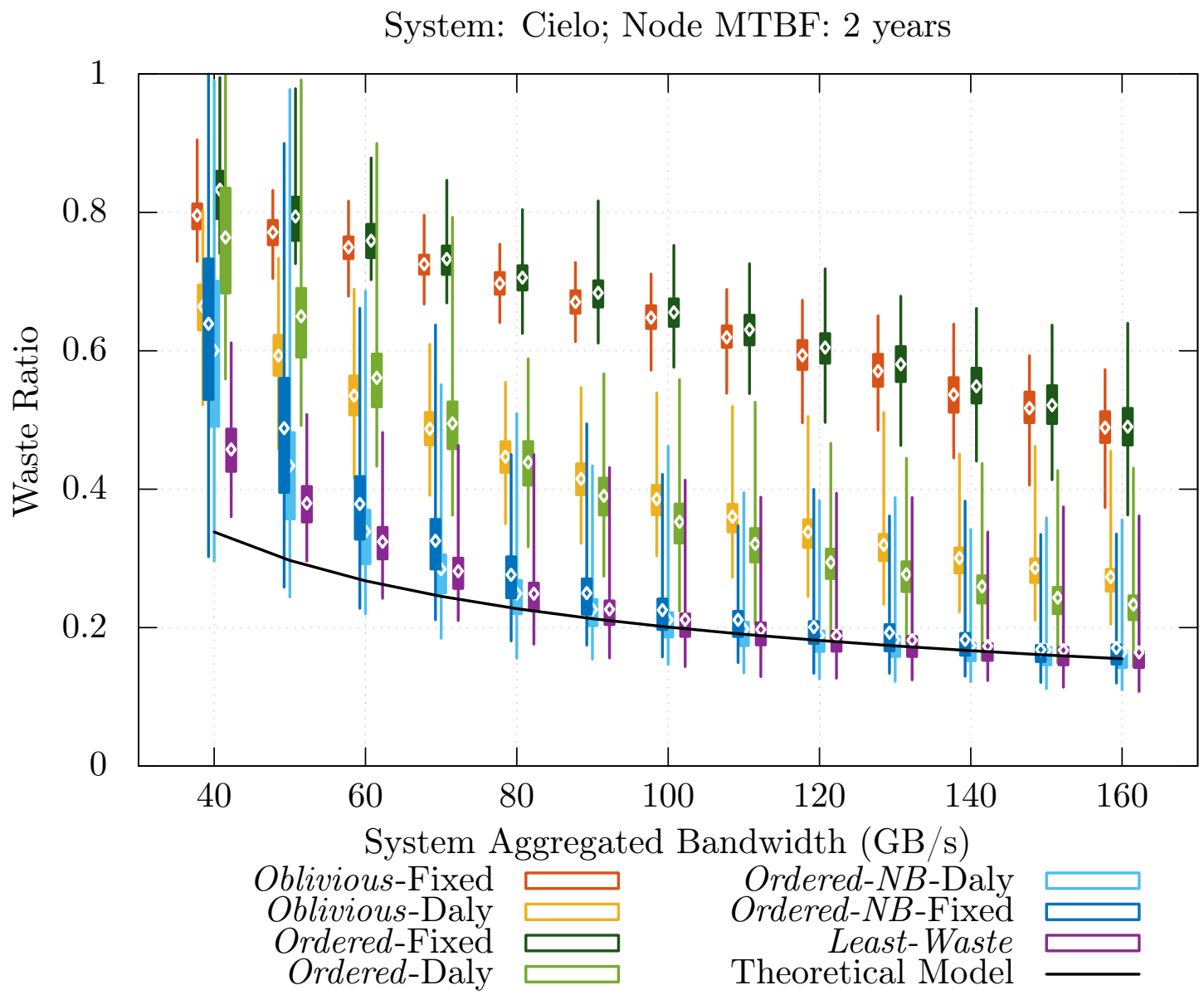

Figure 1: Waste ratio as a function of the system bandwidth for the seven $\mathrm{I} / \mathrm{O}$ and Checkpointing scheduling strategies, and the LANL workload on Cielo.

of each strategy by computing the waste ratio, i.e. the resource waste over a segment of 60 days divided by the application resource usage over that same segment for the baseline simulation. Each simulation is conducted over 1,000 times; the candlestick extremes represent the first and last decile of the measures, while the boxes represent the first and last quartile, and the center the mean value.

The Impact of Available System Bandwidth First, we explore the performance of each approach in a failure-prone environment. Figure 1 represents the waste ratio on Cielo, assuming the node MTBF $\mu_{\text {ind }}$ of 2 years (i.e. a system MTBF of $1 \mathrm{~h}$ ). We vary the filesystem bandwidth from $40 \mathrm{~GB} / \mathrm{s}$ to $160 \mathrm{~GB} / \mathrm{s}$ in order to evaluate the impact of this parameter. We observe three classes of behavior: Oblivious-Fixed and Ordered-Fixed exhibit a waste ratio that decreases as the bandwidth increases, but remains above $40 \%$ even at the maximum theoretical I/O bandwidth; Ordered-NB-Daly, Ordered-NB-Fixed, and Least-Waste quickly decrease to below $20 \%$ of waste, and reach the theoretical model performance ${ }^{6}$; and Oblivious-Daly and Ordered-Daly start at the same level of efficiency as Oblivious-Fixed and Ordered-Fixed, and slowly reach $20 \%$ of waste as the bandwidth increases. Note, in some cases the error bars dip below the theoretical lower

\footnotetext{
${ }^{6}$ Maple code to compute the performance predicted by the theoretical model is available at https://github. com/SMURFSorg/InterferingCheckpoints.
} 
bound. In the simulations, failures have an exponential probability distribution centered around the desired MTBF. For some runs, a lower number of failures experienced during the simulation results in a larger MTBF than the average used in the lower-bound formula; such instances can experience a waste lower than the theoretical model.

This figure shows that with a high frequency of failures, providing each job with the appropriate checkpoint interval is paramount to preventing unnecessary (or even detrimental) checkpoints: the two strategies that render high waste despite high bandwidth rely on a fixed $1 \mathrm{~h}$ interval. However, it also shows that this is not the sole criteria that should be taken into account, nor a necessary condition to extract performance. Even with favorable bandwidth, Oblivious-Daly and OrderedDaly experience nearly twice the waste of the other strategies with same checkpointing period. All strategies that decouple the execution of the application from the filesystem availability (Ordered$N B$-Daly, Ordered-NB-Fixed, Least-Waste) exhibit considerably better performance despite low bandwidth.

Notably, Least-Waste remains the most efficient technique in this study, and reaches the theoretical performance given by Equation (7) for steady-state analysis. This illustrates the efficiency of the proposed heuristic (Equations (1) and (2)) to schedule checkpoints and I/O in a way that avoids interferences, allowing the system to behave as if no interference is experienced, in most cases. The high variation shows that a minority of the runs experienced a significantly higher waste, but such is the case for all algorithms.

The Impact of System Reliability Next, we explore the performance of each approach under low bandwidth (and thus high probability of interference). A scenario with such low bandwidth is not unrealistic. As shown in Luu et al [6], practical bandwidth can be considerably lower than theoretical. Figure 2 represents the waste ratio on Cielo, assuming the aggregated filesystem bandwidth of the system is $40 \mathrm{~GB} / \mathrm{s}$. We vary the node MTBF $\mu_{\text {ind }}$ from 2 years $(1 \mathrm{~h}$ of system MTBF) to 50 years (24h of system MTBF) in order to evaluate the impact of this parameter. Similar to Figure 1, we observe three classes of behavior: Oblivious-Fixed and Ordered-Fixed exhibit a waste ratio that remains constant around $80 \%$ for all values of the MTBF. These approaches are critically dependent on the filesystem bandwidth, and a lower frequency of failures does not significantly improve their performance. The I/O subsystem is saturated, and the applications spends most of their time waiting for it. Oblivious-Daly and Ordered-Daly, see poor efficiency for small MTBF values, but steadily improve to come close to the theoretical bound for higher MTBF values. Lastly, Ordered-NB-Daly, Ordered-NB-Fixed, and Least-Waste quickly reach the theoretical model performance, even with a low MTBF (4 year node MTBF or $2 \mathrm{~h}$ of system MTBF).

For all the strategies that use the Daly checkpointing period, increasing the MTBF reduces the amount of I/O required and thus relieves the pressure of a constrained bandwidth. All strategies that schedule the bandwidth are successful at increasing the efficiency close to the theoretical model. Similarly, Ordered-NB-Fixed, despite its fixed checkpoint interval is capable of reaching a performance comparable to the Daly-based strategies (which reduce the number of total checkpoints). The rapid improvement of the Ordered-NB-Fixed approach can be explained by a combination of 2 factors. Foremost, the non-blocking aspect of the checkpoint provide the I/O subsystem with enough flexibility to order the checkpoint without imposing an additional wait. Delayed checkpoints only translate in additional waste if that application itself is subject to failure. Additionally, for lower MTBFs, the more frequent restarts of interfering jobs, despite the fact that they delay the checkpointing operation, do not introduce additional waste.

\subsection{Evaluating a Prospective System}

In order to understand the impact of the $\mathrm{I} / \mathrm{O}$ contention on future platforms, we use our simulator to explore a prospective system and assess the impact of $\mathrm{I} / \mathrm{O}$ and checkpoint scheduling when the problem size and the machine size will increase. We consider a future system with $7 \mathrm{~PB}$ of main memory and 50,000 compute nodes (e.g. Aurora $\left.{ }^{7}\right)$. Based on the APEX workflow report, we

${ }^{7}$ https://aurora.alcf.anl.gov/ 
System: Cielo; System Aggregated Bandwidth: 40GB/s

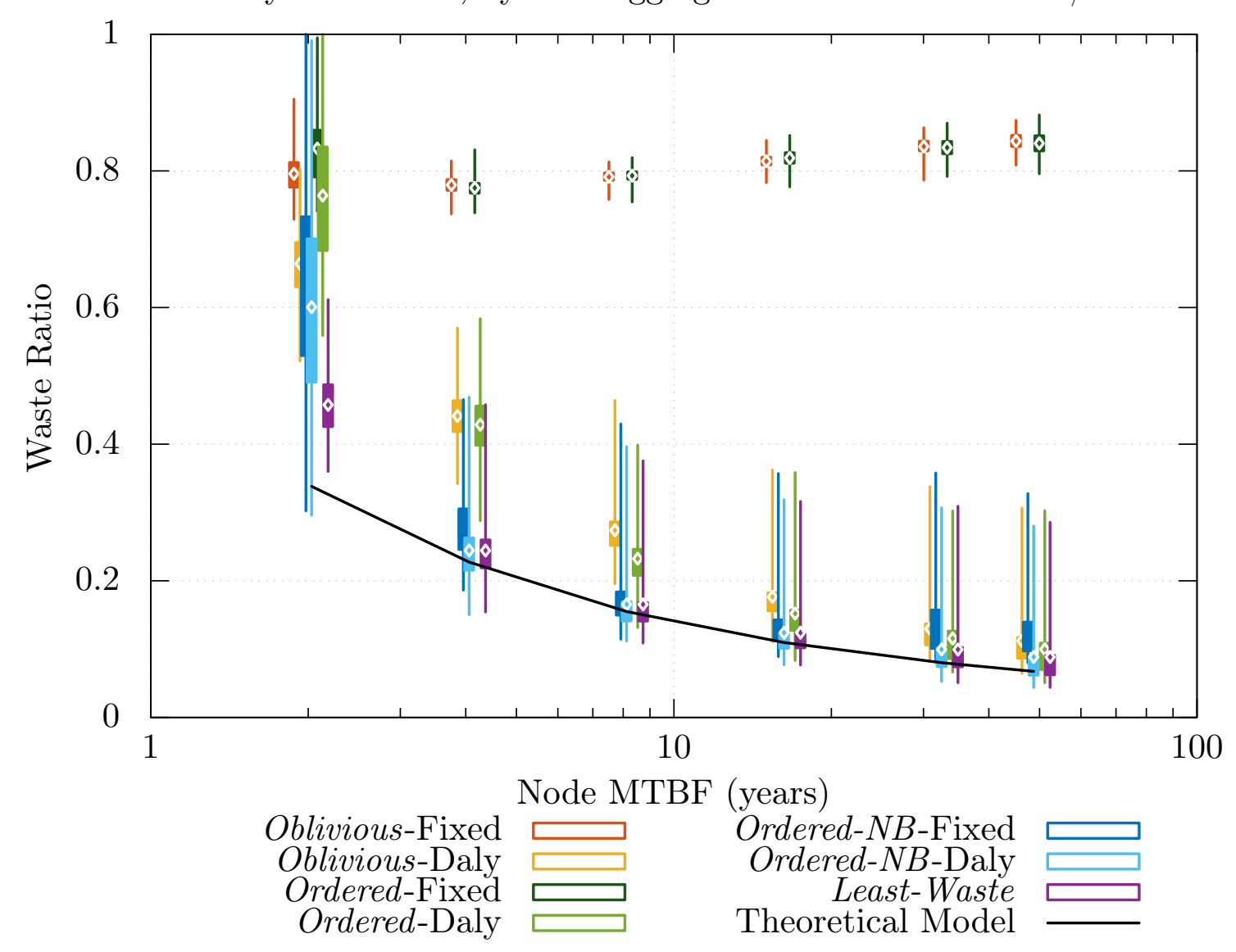

Figure 2: Waste ratio as a function of the system MTBF for the seven I/O and Checkpointing scheduling strategies, and the LANL workload on Cielo. 


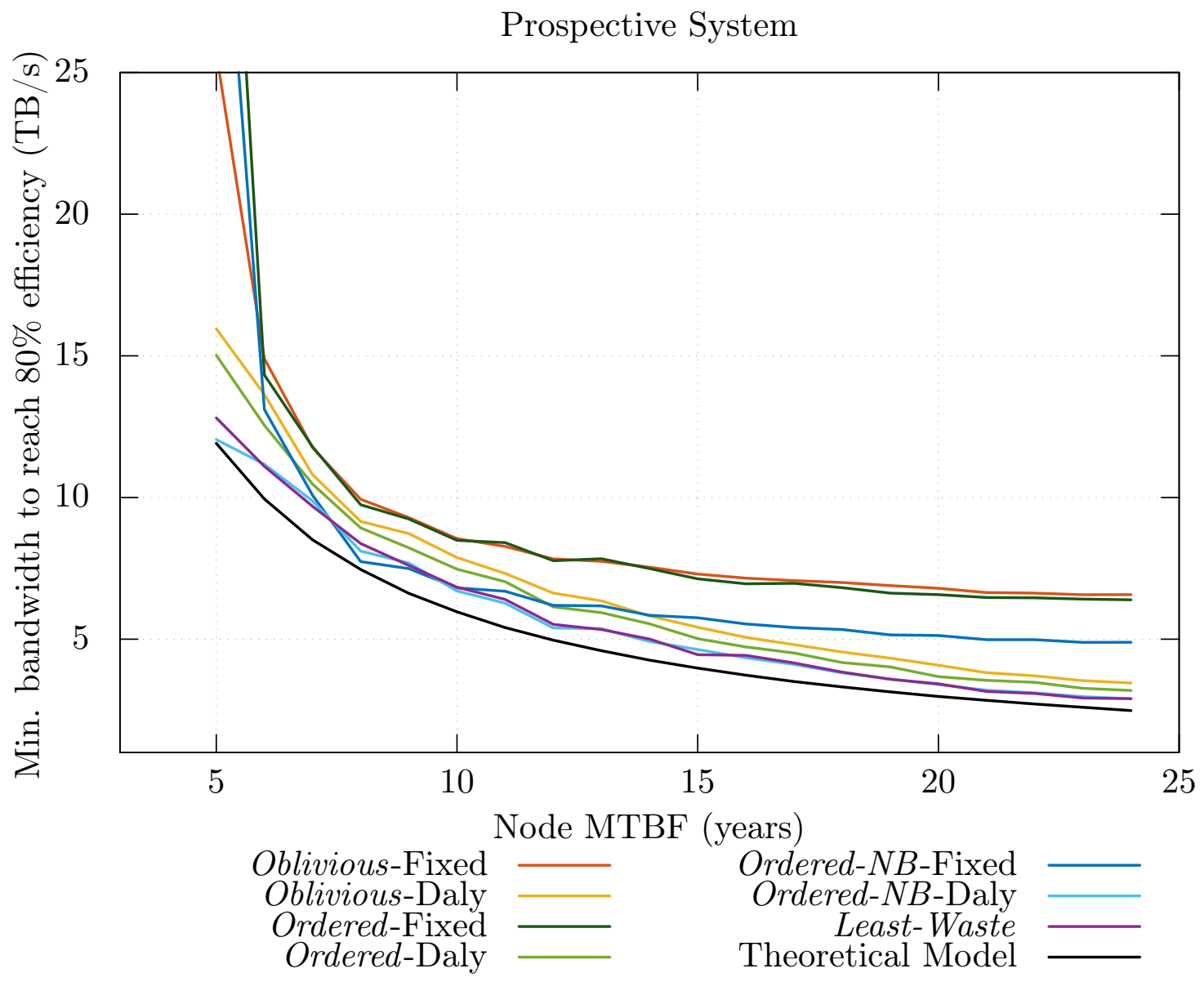

Figure 3: Minimum aggregated filesystem bandwidth to reach $80 \%$ efficiency with the different approaches on the prospective future system. 
extrapolate the increase in problem size expected for the application classes considered previously, and project these applications on the prospective system. We simulate the workload of Table 1, scaling the problem size proportionally to the change in machine memory size. The waste is computed, as previously, by dividing the amount of resource used for checkpoints and lost due to failures by the amount of resource used in a fault-free and resilience-free run with the same initial conditions. We vary system MTBF; and for each strategy, we find the required aggregated practical bandwidth necessary to provide a sustained $80 \%$ efficiency of the system. This $80 \%$ target efficiency is viewed by many programs (e.g. The Exascale Computing Project ${ }^{8}$ ) as a reasonable cost for resilience activities. Figure 3 shows the impact of MTBF and strategies on this prospective system.

When failures are frequent (less than 10 year node MTBF), the most critical element is to reduce the I/O pressure: all strategies that use a fixed and frequent checkpoint interval require greater available bandwidth to reach the target efficiency. In this case, strategies that combine an optimal checkpointing period with $\mathrm{I} / \mathrm{O}$ and checkpoint scheduling (Least-Waste and OrderedNB-Daly) perform similarly, consistently better than all other approaches. These two approaches exhibit a strong resilience to failures, with a bandwidth requirement that only increases by a factor of three between a very unstable system (less than one hour system MTBF), and a stable one (an 8 hour system MTBF). In contrast, the other strategies are much more dependent upon the frequency of failures; the Oblivious-Fixed strategy requires up to 50 times the bandwidth of Least-Waste to reach the same efficiency.

When failures are not endemic (i.e. a node MTBF is at least 15 years and a system MTBF of 2.6 hours), the hierarchy of different approaches stabilizes. The two blocking strategies relying on frequent checkpoints (Oblivious-Fixed and Ordered-Fixed) remain expensive, requiring the highest bandwidth to reach the target efficiency. The next contender, Ordered-NB-Fixed, requires a quarter of the bandwidth to reach the same efficiency. Despite using the same fixed checkpoint interval as the previous methods, it benefits from not blocking when the filesystem is not available. This is sufficient, when failures are rare, to obtain a significant performance gain. All Daly-based strategies benefit from reduced I/O pressure, and reach the target efficiency with around half the bandwidth needed by Oblivious-Fixed. We also observe that Ordered-NB-Daly and Least-Waste remain the most efficient strategies for the whole MTBF spectrum. These results highlight that checkpoint-based strategies can scale to satisfy the need of future platforms, whether by integrating $\mathrm{I} / \mathrm{O}$-aware scheduling strategies or by significantly over-provisioning the I/O partition.

\section{$7 \quad$ Related Work}

We first discuss research regarding checkpoint-induced I/O pressure, followed by works that regard avoiding I/O interference. These techniques are not necessarily independent: generally, reducing $\mathrm{I} / \mathrm{O}$ pressure will reduce the likelihood of interference. Therefore, we focus our I/O interference discussion to those techniques which consider the global scheduling of checkpoints and/or application $\mathrm{I} / \mathrm{O}$ across a platform.

Checkpointing and I/O For a single application, the Young/Daly formula [3, 4] gives the optimal checkpointing period. This period minimizes platform waste, defined as the fraction of job execution time that does not contribute to its progress. The two sources of waste are the time spent taking checkpoints (which motivates longer checkpoint periods) and the time needed to recover and re-execute after each failure (which motivates shorter checkpoint periods). The Young/Daly period achieves the optimal trade-off between these sources to minimize the total waste. Arunagiri et al. [12] studied longer, sub-optimal periods with the intent of reducing I/O pressure and showed, both analytically and empircally using four real platforms, that a decrease in the $\mathrm{I} / \mathrm{O}$ requirement can be achieved with only a small increase in waste.

\footnotetext{
${ }^{8}$ https://exascaleproject.org
} 
Reducing I/O Pressure There are two general strategies for reducing I/O pressure from a single application: hiding or reducing checkpoint commit times without reducing checkpoint data volumes, and reducing commit times by reducing checkpoint data volumes. Strategies that attempt to hide checkpoint times include Diskless [13] and remote checkpoint protocols [14] which leverage the typically higher available bandwidths of the network or other storage media like RAM in order to mitigate the performance of slower storage media like spinning or solid-state disks. Additionally, remotely stored checkpoints have the additional benefit of allowing systems to survive non-transient node failures. Similarly, multi-level checkpoint protocols like SCR $[9,15]$ attempt to hide checkpoint commit times by writing checkpoints to RAM, flash storage, or local disk on the compute nodes [16] in addition to the parallel file system thereby improving checkpoint or general I/O bandwidth. Finally, checkpoint-specific file systems like PLFS [17] leverage the I/O patterns and characteristics specific to checkpoint data to optimize checkpoint data transfers to/from parallel file systems and therefore reduce checkpoint commit times.

Strategies that attempt to reduce checkpoint sizes include memory exclusion, which leverage user-directives or other hints to exclude portions of process address spaces from checkpoints [18]. Additionally, incremental checkpointing protocols reduce checkpoint volumes by utilizing the OS's memory page protection facilities to detect and save only pages that have been updated between consecutive checkpoints $[19,20,21,22,23,24,25]$. Similarly, page-based hashing techniques can also be used to avoid checkpointing pages that have been written to but whose content has not changed [26]. Finally, compression-based techniques use standard compression algorithms to reduce checkpoint volumes [27] and can be used at the compiler-level [28] or in-memory [29]. Related, Plank et al. proposed differential compression to reduce checkpoint sizes for incremental checkpoints [30] and Tanzima et al. show that similarities amongst checkpoint data from different processes can be exploited to compress and reduce checkpoint data volumes [31]. Finally, Sasaki et al propose a lossy compression method based on wavelet transform and vector quantization to the checkpoints of a production climate application [32], while Ni et al [33] study the trade-offs between the loss of precision, compression ratio, and application correctness due to lossy compression.

Avoiding I/O interference Most closely related to our work, a number of studies have considered the global scheduling of checkpoints and other I/O across a platform to reduce overall congestion, thereby increasing performance. Aupy et al. [34] presented a decentralized I/O scheduling technique for minimizing the congestion due to checkpoint interference by taking advantage of the observed periodic and deterministic nature of HPC application checkpoints and I/O. This technique allows the job scheduler to pre-define each application's I/O behavior for their entire execution. Similarly, a number of works have investigated the efficiency of online schedulers for data intensive $[35,36]$ and HPC workload I/O [8, 37, 38, 39]. Finally, a number of works have investigated utilizing recorded system reliability information [40] and the statistical properties of these failures [41] to determine effective checkpoint intervals for the portion of the system used by the workload.

Summary We distinguish our work from these previous studies in a number of important ways. First, unlike a number of the previous studies, our technique considers existing non-CR application $\mathrm{I} / \mathrm{O}$. Additionally, our approach is agnostic to the $\mathrm{I} / \mathrm{O}$ patterns of the considered applications as long as they are known. Also, we attempt to optimize the efficiency of the entire platform, with the changing workloads and failures running on that platform, rather than just considering one workload. Finally and most importantly, this approach provides optimal checkpointing periods in environments where I/O is highly constrained and Daly/Young's formula is less appropriate, a common scenario on many leadership-class systems.

\section{Conclusion and Future Work}

As we design larger, likely more error-prone, platforms, effectively protecting applications from platform faults becomes critical. Current fault-protection techniques available on production plat- 
forms rely on checkpoint/restart to ensure fault protection. However, these techniques, by their very nature, regularly save the application state to stable storage, and therefore increase the burden of the already overtaxed I/O subsystem.

Considering a comprehensive I/O interference model for platforms susceptible to I/O contention, we designed multiple $\mathrm{I} / \mathrm{O}$ scheduling algorithms that target improving overall platform job throughput via waste minimization. We also theorized a lower-bound for platform waste for $\mathrm{I} / \mathrm{O}$ constrained checkpointing workloads. We use this theoretical lower-bound to demonstrate the effectiveness of our Least-Waste I/O scheduling and to compare its performance with other I/O scheduling strategies. Our strategy invariably outperforms the others with respect to the platform efficiency. Unsurprisingly, the biggest gains are rendered on the platforms with a lowest MTBF or greater degrees of under-provisioned I/O. Through simulation, we also show a path to supporting $\mathrm{C} / \mathrm{R}$ on a prospective system while maintaining $80 \%$ platform efficiency, all without a large investment in the I/O subsystem.

As burst-buffers and other NVRAM storage mechanisms become more common, a natural extension of this work would consider their impact on $\mathrm{I} / \mathrm{O}$ contention/interference. Increasing the available I/O bandwidth leads to reduced waste (due to the decrease in checkpoint duration but also an increase in the optimal checkpoint frequency and therefore a decrease in the restart time), while providing relief to the shared I/O subsystem to better absorb additional checkpoint information. We speculate that scheduling parallel filesystem $\mathrm{I} / \mathrm{O}$ with a heuristic that prioritizes jobs to minimize failure impact can help to improve overall burst-buffer efficiencies. Such a heuristic would build upon the strategies discussed in this work and extend them to the new framework.

\section{References}

[1] O. Weidner, M. Atkinson, A. Barker, and R. Filgueira Vicente, "Rethinking high performance computing platforms: Challenges, opportunities and recommendations," in Proc. DataIntensive Distributed Computing DIDC. ACM, 2016.

[2] A. Bhatele, K. Mohror, S. H. Langer, and K. E. Isaacs, "There goes the neighborhood: Performance degradation due to nearby jobs," in Proc. Supercomputing (SC '13). ACM, 2013.

[3] J. W. Young, "A first order approximation to the optimum checkpoint interval," Comm. of the $A C M$, vol. 17 , no. 9, pp. 530-531, 1974.

[4] J. T. Daly, "A higher order estimate of the optimum checkpoint interval for restart dumps," FGCS, vol. 22, no. 3, pp. 303-312, 2006.

[5] T. Herault and Y. Robert, Eds., Fault-Tolerance Techniques for High-Performance Computing, ser. Computer Communications and Networks. Springer Verlag, 2016.

[6] H. Luu, M. Winslett, W. Gropp, R. Ross, P. Carns, K. Harms, M. Prabhat, S. Byna, and Y. Yao, "A multiplatform study of I/O behavior on Petascale supercomputers," in Proc. 24th Int. Symp. on High-Performance Parallel and Distributed Computing (HPDC'15). ACM, 2015 .

[7] APEX, "APEX Workflows," LANL, NERSC, SNL, Research report SAND2016-2371 and LAUR-15-29113, 2016.

[8] M. Dorier, G. Antoniu, R. Ross, D. Kimpe, and S. Ibrahim, "CALCioM: Mitigating I/O interference in HPC systems through cross-application coordination," in IEEE Int. Parallel and Distributed Processing Symposium (IPDPS). IEEE Computer Society, 2015, pp. 156-163.

[9] A. Moody, G. Bronevetsky, K. Mohror, and B. R. de Supinski, "Design, Modeling, and Evaluation of a Scalable Multi-level Checkpointing System," in Proc. Supercomputing (SC '10). ACM, 2010. 
[10] L. Bautista-Gomez, S. Tsuboi, D. Komatitsch, F. Cappello, N. Maruyama, and S. Matsuoka, "FTI: High performance fault tolerance interface for hybrid systems," in Proc. SC'11, 2011.

[11] S. Boyd and L. Vandenberghe, Convex Optimization. Cambridge University Press, 2004.

[12] S. Arunagiri, J. T. Daly, and P. J. Teller, "Modeling and Analysis of Checkpoint I/O Operations," in Analytical and Stochastic Modeling Techniques and Applications: 17th International Conference. Springer, 2010, pp. 387-399.

[13] J. Plank, K. Li, and M. Puening, "Diskless checkpointing," IEEE Trans. Parallel and Distributed Systems, vol. 9, no. 10, pp. 972-986, oct 1998.

[14] J. Cornwell and A. Kongmunvattana, "Efficient System-Level Remote Checkpointing Technique for BLCR," in Eighth Int. Conf. on Information Technology: New Generations (ITNG), 2011, pp. 1002-1007.

[15] N. H. Vaidya, "A case for two-level distributed recovery schemes," in ACM SIGMETRICS Joint Int. Conf. on Measurement and Modeling of Computer Systems. ACM, 1995.

[16] A. Kougkas, M. Dorier, R. Latham, R. Ross, and X. H. Sun, "Leveraging burst buffer coordination to prevent I/O interference," in IEEE 13th Int. Conf. on e-Science (e-Science). IEEE, 2017, pp. 372-379.

[17] J. Bent, G. Gibson, G. Grider, B. McClelland, P. Nowoczynski, J. Nunez, M. Polte, and M. Wingate, "PLFS: a checkpoint filesystem for parallel applications," in Conference on High Performance Computing Networking, Storage and Analysis (SC '09), 2009, pp. 21:1-21:12.

[18] J. S. Plank, Y. Chen, K. Li, M. Beck, and G. Kingsley, "Memory Exclusion: Optimizing the Performance of Checkpointing Systems," Software - Practice 85 Experience, vol. 29, no. 2, pp. 125-142, 1999.

[19] G. Bronevetsky, D. Marques, K. Pingali, S. McKee, and R. Rugina, "Compiler-enhanced incremental checkpointing for OpenMP applications," in IEEE International Symposium on Parallel\&Distributed Processing, 2009, pp. 1-12.

[20] Y. Chen, K. Li, and J. S. Plank, "CLIP: A Checkpointing Tool for Message-passing Parallel Programs," in SuperComputing '97, San Jose, CA, 1997. [Online]. Available: http://citeseer.ist.psu.edu/chen97clip.html

[21] E. N. Elnozahy, D. B. Johnson, and W. Zwaenpoel, "The Performance of Consistent Checkpointing," in 11th IEEE Symposium on Reliable Distributed Systems, Houston, TX, 1992.

[22] K. Li, J. F. Naughton, and J. S. Plank, "Low-Latency, Concurrent Checkpointing for Parallel Programs," IEEE Transactions on Parallel and Distributed Systems, vol. 5, no. 8, pp. 874-879, August 1994.

[23] J. S. Plank, M. Beck, G. Kingsley, and K. Li, "Libckpt: Transparent Checkpointing under Unix," in USENIX Winter 1995 Technical Conference, New Orleans, LA, January 1995, pp. $213-224$.

[24] M. Paun, N. Naksinehaboon, R. Nassar, C. Leangsuksun, S. L. Scott, and N. Taerat, "Incremental Checkpoint Schemes for Weibull Failure Distribution," International Journal of Computer Science, vol. 21, no. 3, pp. 329-344, 2010.

[25] S. Al-Kiswany, M. Ripeanu, S. Vazhkudai, and A. Gharaibeh, "stdchk: A Checkpoint Storage System for Desktop Grid Computing," in Distributed Computing Systems, 2008. ICDCS '08. The 28th International Conference on, june 2008, pp. 613-624. 
[26] K. B. Ferreira, R. Riesen, R. Brightwell, P. G. Bridges, and D. Arnold, "Libhashckpt: Hashbased Incremental Checkpointing Using GPUs," in Proceedings of the 18th EuroMPI Conference, September 2011.

[27] D. Ibtesham, D. Arnold, P. G. Bridges, K. B. Ferreira, and R. Brightwell, "On the viability of compression for reducing the overheads of checkpoint/restart-based fault tolerance," 2012 41st International Conference on Parallel Processing, vol. 0, pp. 148-157, 2012.

[28] C.-C. Li and W. Fuchs, "CATCH-compiler-assisted techniques for checkpointing," in FaultTolerant Computing, 1990. FTCS-20. Digest of Papers., 20th International Symposium, jun 1990, pp. 74-81.

[29] J. S. Plank and K. Li, "ickp: A Consistent Checkpointer for Multicomputers," Parallel \& Distributed Technology: Systems \& Applications, IEEE, vol. 2, no. 2, pp. 62-67, 1994.

[30] J. S. Plank, J. Xu, and R. H. B. Netzer, "Compressed Differences: An Algorithm for Fast Incremental Checkpointing," University of Tennessee, Tech. Rep. CS-95-302, August 1995. [Online]. Available: http://web.eecs.utk.edu/ plank/plank/papers/CS-95-302.html

[31] T. Z. Islam, K. Mohror, S. Bagchi, A. Moody, B. De Supinski, and R. Eigenmann, "MCRENGINE: A Scalable Checkpointing System Using Data-Aware Aggregation and Compression," in Proc. Supercomputing (SC'12). ACM, 2012.

[32] N. Sasaki, K. Sato, T. Endo, and S. Matsuoka, "Exploration of lossy compression for application-level checkpoint/restart," in IPDPS. IEEE, 2015.

[33] X. Ni, E. Meneses, N. Jain, and L. V. Kalé, "ACR: Automatic Checkpoint/Restart for Soft and Hard Error Protection," in Proc. SC'14. ACM, 2014.

[34] G. Aupy, A. Gainaru, and V. Le Fevre, "Periodic I/O Scheduling for Super-computers," INRIA, Research report RR-9037, Feb. 2017.

[35] S. Groot, K. Goda, D. Yokoyama, M. Nakano, and M. Kitsuregawa, "Modeling I/O Interference for Data Intensive Distributed Applications," in Proc. 8th Annual ACM Symp. on Applied Computing (SAC). ACM, 2013, pp. 343-350.

[36] H. Sim, Y. Kim, S. S. Vazhkudai, D. Tiwari, A. Anwar, A. R. Butt, and L. Ramakrishnan, "AnalyzeThis: an analysis workflow-aware storage system," in Proc. Supercomputing (SC'15). ACM, 2015.

[37] A. Gainaru, G. Aupy, A. Benoit, F. Cappello, Y. Robert, and M. Snir, "Scheduling the I/O of HPC Applications Under Congestion," in IEEE IPDPS. IEEE, 2016.

[38] Z. Zhou, X. Yang, D. Zhao, P. Rich, W. Tang, J. Wang, and Z. Lan, "I/O-Aware Batch Scheduling for Petascale Computing Systems," in IEEE International Conference on Cluster Computing, Sept 2015, pp. 254-263.

[39] S. Herbein, D. H. Ahn, D. Lipari, T. R. Scogland, M. Stearman, M. Grondona, J. Garlick, B. Springmeyer, and M. Taufer, "Scalable I/O-Aware Job Scheduling for Burst Buffer Enabled HPC Clusters," in Proceedings HPDC. ACM, 2017, pp. 70-79.

[40] A. J. Oliner, L. Rudolph, and R. K. Sahoo, "Cooperative checkpointing: A robust approach to large-scale systems reliability," in Proceedings of the 20th Annual International Conference on Supercomputing, ser. ICS '06. New York, NY, USA: ACM, 2006, pp. 14-23.

[41] D. Tiwari, S. Gupta, and S. S. Vazhkudai, "Lazy checkpointing: Exploiting temporal locality in failures to mitigate checkpointing overheads on extreme-scale systems," in Dependable Systems and Networks (DSN), 44th Annual IEEE/IFIP International Conference on, 2014, p. $25-36$. 


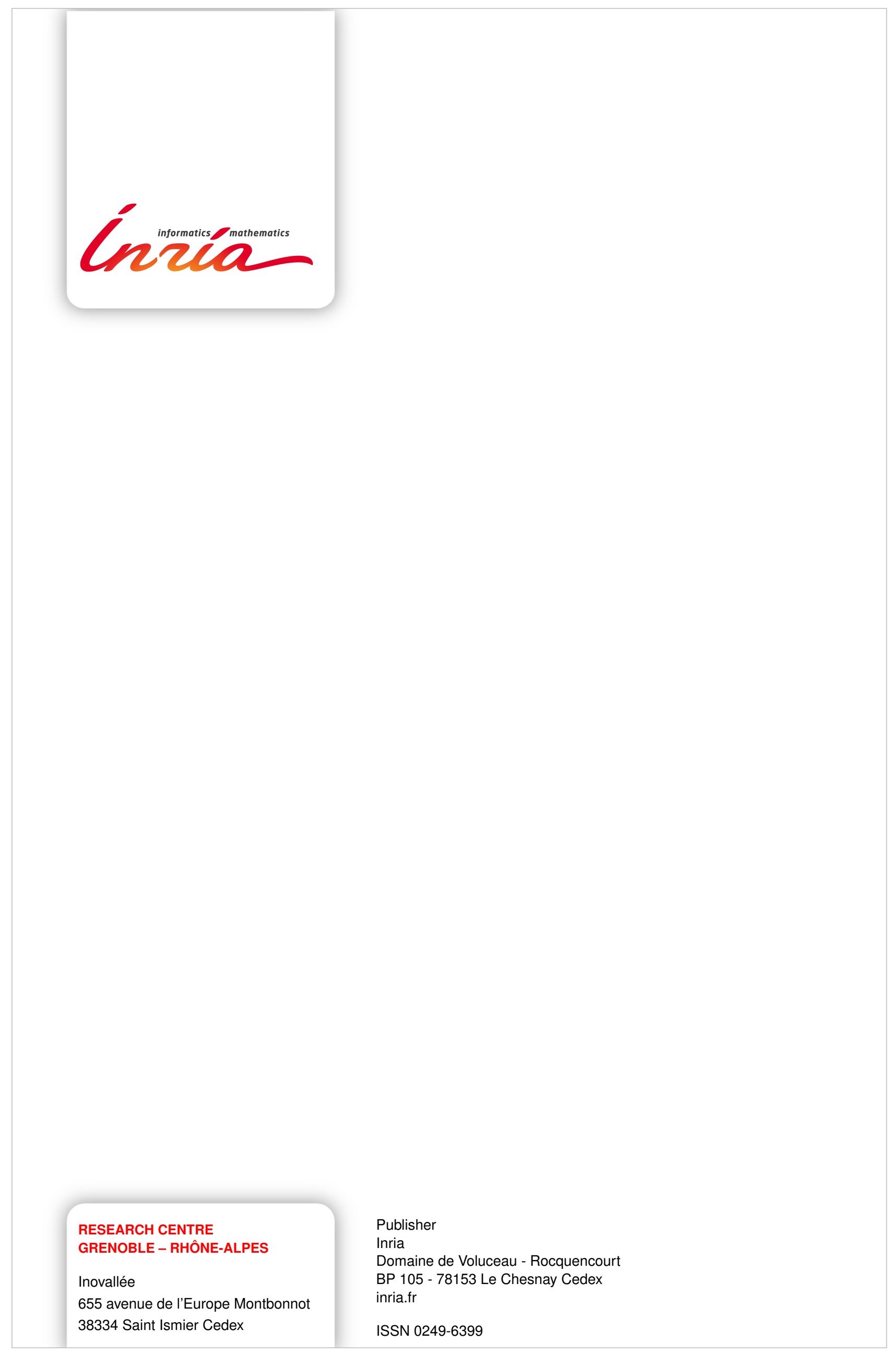

